\title{
Editorial
}

\section{Practical Considerations for COVID-19 Vaccine Design and Development}

\author{
Md. Azizul Haque ${ }^{1}$
}

As of September 24, 2020, SARS-CoV-2 pandemic has spread to 213 countries around the world, has a confirmed case number of 32,097,561 and caused a death toll of 981,968 and counting. ${ }^{1}$ Although transmission-mitigation strategies has been found to be partially effective in controlling the transmission of the virus, relentless progression of this highly infectious virus can be most effectively ensured by mass vaccination of the world population. Scientists throughout the world are trying their best to develop a vaccine in an accelerated timeframe with 38 vaccine candidates currently in clinical development, as of $22^{\text {nd }}$ September, 2020. ${ }^{2}$ To meet the urgent need for a vaccine, a new pandemic vaccine development paradigm has been proposed that compresses the development timeline from 10-15 years to 1-2 years.

Coronaviruses have a large genome of 26-32 kb with an overall similarity in the organization of the genome. In the 5 end of the genome, two open reading frames (ORF) encode for non-structural proteins, while the 3 end encodes for the Spike protein (S), envelope protein (E), membrane protein $(\mathrm{M})$, and nucleocapsid $(\mathrm{N}){ }^{3}{ }^{3}$ The initial site of infection of SARS-CoV-2 is the respiratory tract. On entry, SARS-CoV-2 interacts with the angiotensin- converting enzyme 2 (ACE2) receptor on bronchial and alveolar epithelial cells through its spike (S) protein receptor- binding domain (RBD), which is subsequently primed by a specific cellular serine protease, transmembrane protease serine 2 (TMPRSS2), to gain entry. ${ }^{4}$
The six major types of candidate vaccine for coronavirus disease 2019 (COVID-19) include: recombinant viral vectored, inactivated virus, protein subunit, virus- like particles, nucleic acid based and live attenuated virus. ${ }^{2}$ The $S$ protein has been proposed as one of the most promising candidates for vaccine design for the SARS-CoV2 because of its ability to induce neutralizing antibodies and a strong $\mathrm{T}$ cell response. The $\mathrm{S}$ glycoprotein is divided into two subunits, S1 and S2, where S1 contains the receptor-binding domain (RBD), including smaller receptor-binding motif (RBM), while the fusion protein is located in S2. Both SARS-CoV and SARS-CoV-2 bind to the angiotensin-converting enzyme 2 (ACE-2) as the primary target, whereas the predominant cellular receptor for MERS is dipeptidyl peptidase 4 (DPP4). ${ }^{5,6}$ While the S protein is by far the most studied region for vaccine designs, in silico analyses of potential immunogenic epitopes in the SARS-CoV-2 have suggested several domains within the N, M, and E protein as well as the nonstructural proteins primarily focusing on $\mathrm{T}$ cell responses. ${ }^{7} \mathrm{~T}$ cells, especially $\mathrm{CD} 4+$ and $\mathrm{CD} 8+$, are crucial in eliciting a specific and adequate immune response and producing a long-term immunological memory. A concise review by Grifoni et al. shed light on the importance of T cell response in COVID-19 and its potential in developing a new vaccine. Essentially, they looked up the antigens that the virus-specific $\mathrm{T}$ cells reacted to in exposed COVID-19 patients and compared them to healthy individuals. The team 
found that $100 \%$ of the exposed patients had $\mathrm{CD} 4+\mathrm{T}$ cells that responded to the $\mathrm{S}$ protein, $\mathrm{N}$ protein, and $\mathrm{M}$ protein. This is an interesting finding as adding the $\mathrm{M}$ protein and the $\mathrm{N}$ protein to the vaccines with the $\mathrm{S}$ protein increases the chance of mimicking a natural COVID-19 infection, thereby eliciting a better immune response in the case of a potential future infection. ${ }^{8}$ We do not yet understand the durability of the antibody responses to SARS-CoV-2. However, previous longitudinal studies of patients with SARS-CoV infection reported that neutralizing antibody titers diminished substantially between 1 year and 2 years after infection. ${ }^{9}$ Whereas the current successful human antiviral vaccines, such as influenza and measles vaccines, depend largely on the induction of antibody responses, emerging evidence suggests that, effective protection against SARS-CoV-2 requires both antibody- mediated and $\mathrm{T}$-cell mediated immunity. It is well known that CD4+ T cell help is important for optimal antibody responses and for CD8+ $\mathrm{T}$ cell activation in host defense. Furthermore, cytotoxic CD8+ T cells are crucial for viral clearance if neutralizing antibodymediated protection is incomplete. In the elderly population, T cell-mediated immunity generally is a more reliable correlate of vaccine protection than antibody titers, strongly support the inclusion of $\mathrm{T}$ cell responses in COVID-19 vaccine design.

Four vaccine developers are running phase 3 trials of COVID-19 vaccine using non-replicating virus vectors: University of Oxford/AstraZeneca is working with ChAdOx1-S, CanSino Biological Inc./Beijing Institute of Biotechnology is working with Adenovirus Type 5 Vector, Gamaleya Research Institute of Russia is using dual adenovirus vector (rAd26-S+rAd5-S), and Janssen Pharmaceutical Companies are using Ad26COVS1. Vaccines of the University of Oxford/AstraZeneca and Cansino Biological Inc./Beijing Institute of Biotechnology require a single intramuscular dose, whereas vaccines of Gamaleya Research Institute of Russia and Janssen Pharmaceutical Companies require two intramuscular doses at 0,21 days and 0,56 days respectively. Inactivated vaccines of three
Chinese institutes have reached the level of phase 3: Sinovac, Wuhan Institute of Biological Products/Sinopharm and Beijing Institute of Biological Products/Sinopharm. All of the inactivated vaccines require two intramuscular doses at 0,14 or 0,21 days. Two RNA vaccines have reached phase 3 trails so far: one by Moderna/NIAID which uses LNP-encapsulated mRNA and another is by BioNTech/Fosun Pharma/Pfizer, which uses 3 LNP-mRNAs. Both of these RNA vaccines require two intramuscular doses at 0,28 days. $^{2}$

Ever since Edward Jenner first developed small vaccine in 1798, dozens of vaccines have saved hundreds of millions of lives. As COVID-19 vaccines are being developed towards an ultimate goal of global mass immunization, vaccine safety is of paramount importance. Any indication of a lack of safety consideration could also fuel the anti-vaccination movement and vaccine hesitancy, which would jeopardize the desired effect of achieving herd immunity. The highly susceptible elderly populations and those with underlying medical conditions are in particular need of highly safe and effective vaccines. The safety of a vaccine is generally determined by the nature of the vaccine platform, the choice of adjuvant, the mode and route of vaccine administration, the age of vaccine recipients and the status of pre- existing vaccine immunity. For example, replicating live attenuated virus or viral- vectored vaccines may not be safe for a respiratory mucosal route of vaccination. Scientists around the world are harnessing global coalition to develop a safe and effective vaccine to fight the deadly COVID-19 pandemic and we hope they will be able to deliver one very soon.

\section{References:}

1. World Health Organization. WHO coronavirus disease (COVID-19) dashboard. WHO https:// covid19. who. int/ (2020).

2. World Health Organization. Draft landscape of COVID-19 candidate vaccines. WHO https:// www. who.int/publications/m/item/draft- landscape-ofcovid- 19-candidate- vaccines (2020).

3. Lu, R.; Zhao, X.; Li, J.; Niu, P.; Yang, B.; Wu, H.; Wang, W.; Song, H.; Huang, B.; Zhu, N.; et al. 
Genomic characterisation and epidemiology of 2019 novel coronavirus: Implications for virus origins and receptor binding. Lancet 2020; 395:565-574.

4. Hoffmann, M. et al. SARS- CoV-2 cell entry depends on ACE2 and TMPRSS2 and is blocked by a clinically proven protease inhibitor. Cell. 2020; 181:271-280.

5. Chu, H.; Chan, J.F.-W.; Yuen, T.T.-T.; Shuai, H.; Yuan, S.; Wang, Y.; Hu, B.; Yip, C.C.-Y.; Tsang, J.O.-L.; Huang, X.; et al. Comparative tropism, replication kinetics, and cell damage profiling of SARS-CoV-2 and SARS-CoV with implications for clinical manifestations, transmissibility, and laboratory studies of COVID-19: An observational study. Lancet Microbe 2020; 1:14-23.
6. Du, L.; He, Y.; Zhou, Y.; Liu, S.; Zheng, B.-J.; Jiang, S. The spike protein of SARS-CoV-A target for vaccine and therapeutic development. Nat. Rev. Genet. 2009, 7, 226-236.

7. Lee, C.H.; Koohy, H. In silico identification of vaccine targets for 2019-n CoV. F1000 Research 2020; 9:145.

8. Grifoni et al., Cell 2020; 181:1489-1501.

9. Cao, W.-C., Liu, W., Zhang, P.-H., Zhang, F. \& Richardus, J. H. Disappearance of antibodies to SARS- associated coronavirus after recovery. N. Engl. J. Med. 2207; 357, 1162-1163.

10. Tay, M. Z., Poh, C. M., Rénia, L., MacAry, P. A. \& $\mathrm{Ng}, \mathrm{L}$. F. P. The trinity of COVID-19: immunity inflammation and intervention. Nat. Rev. Immunol. 2020; 20, 363-374. 